médecine/sciences $2000 ; 16: 1106-8$

\title{
HNF-6 : \\ pièce maîtresse d'un réseau de facteurs de transcription hépatiques contrôlés par l'hormone de croissance
}

L'hormone de croissance promeut la croissance linéaire des os longs, un phénomène illustré de façon spectaculaire par le nanisme ou le gigantisme qui résultent respectivement d'un défaut ou d'un excès de production d'hormone de croissance. En réalité, cet effet de l'hormone de croissance dépend de son action sur le foie où elle induit la sécrétion d'IGF-I (insulin-like growth factor-I), un médiateur important de l'effet de l'hormone de croissance sur le tissu osseux, et la production de la sousunité acido-labile de l'IGF-I binding protein-3 et de certains cytochromes P450. En outre, l'hormone de croissance modifie le métabolisme hépatique par des mécanismes encore mal connus.

Le mécanisme moléculaire d'action de l'hormone de croissance s'apparente à celui des cytokines: l'hormone se lie à la surface des cellules cibles, sur son récepteur transmembranaire dénué d'activité enzymatique, et provoque sa dimérisation. Puis ce récepteur recrute et active soit la protéine tyrosine kinase JAK2, soit une protéine $\mathrm{G}$ trimérique couplée à la voie de la protéine kinase $\mathrm{C}$, ce qui déclenche des cascades de phosphorylation et de déphosphorylation. Les effets de l'hormone de croissance peuvent être directement liés au changement d'activité des protéines cibles, ou à celui de facteurs de transcription qui modulent

\author{
HNF-6 intervient dans l'action \\ de l'hormone de croissance \\ sur le foie
}

Les travaux de notre laboratoire ont montré qu'un des facteurs de transcription induits par l'hormone de croissance dans le foie est HNF-6 [1]. Ce facteur [2] (m/s 1996, $n^{\circ} 12$, p. 1431-32) est le prototype d'une nouvelle classe, dite ONECUT, de protéines à domaines "cut-homéo » de liaison à l'ADN [3, 4]. Elles se caractérisent par la présence d'un seul domaine cut - alors que la protéine cut de Drosophila et son homologuechez les mammifères en comportent trois - et par un homéo-domaine d'un type particulier. HNF-6 n'est présent que dans quelques tissus, principalement le foie où il contrôle la transcription de gènes codant pour des enzymes du métabolisme glucidique $[2,5]$, des cytochromes P450 [1] et des protéines plasmatiques [3]. HNF-6 stimule également la transcription des gènes codant pour deux autres facteurs de transcription hépatiques, HNF-3 $\beta$ qui appartient à la famille forkhead/winged-helix, et HNF-4 qui appartient à celle des récepteurs nucléaires orphelins [6].

In vivo, dans l'heure qui suit l'injection de l'hormone de croissance, on observe une augmentation du nombre des transcrits de HNF-6, qui atteint son maximum après 3 heures, puis revient à son niveau de base. Des résultats semblables sont observés in vitro après stimulation par l'hormone de croissance d'hépatocytes en culture primaire. Cette action de l'hormone de croissance résulte d'une action rapide et directe sur la transcription du gène hnf6, dont le clonage et la caractérisation du promoteur ont permis de mieux comprendre les mécanismes.

\section{Le contrôle}

de l'expression du gène $h n f 6$

par l'hormone de croissance

On sait que la phosphorylation des récepteurs de l'hormone de croissance par JAK2 permet le recrutement de certains membres de la famille $\operatorname{STAT}\left(\mathrm{m} / \mathrm{s} 1998, n^{\circ} 10\right.$, p. 1129) qui forment des dimères et migrent vers le noyau cellulaire où ils interagissent avec des séquences proches de celles qui active l'interféron $\gamma$ (Gamma interferon-activated sequence-like element) sur leurs gènes cibles. Une telle séquence est trouvée dans le promoteur du gène $h n f 6$, et nos données récentes [7] montrent que l'hormone de croissance provoque le recrutement de STAT5 dont la liaison à cette séquence permet l'activation de la transcription du gène $h n f 6$.

Une autre caractéristique du promoteur du gène $h n f 6$ est la présence d'une séquence de liaison pour HNF4. Nos résultats montrent que HNF-4 se lie bien au promoteur hnf6 et en stimule l'activité. De plus, le traite- 
ment par l'hormone de croissance augmente, par un mécanisme encore inconnu, l'affinité de liaison de HNF4 au promoteur et donc l'activité de celui-ci [7].

Cependant, HNF-6 stimulant l'expression du gène hnf4 et HNF-4 celle du gène $h n f 6$, l'hormone de croissance aurait dû logiquement entraîner une boucle de rétro-action positive caractérisée par une augmentation persistante du transcrit de HNF-6. Or, l'effet stimulateur de l'hormone de croissance sur le gène $h n f 6$ disparaît rapidement, ce qui suggère que cette hormone est aussi à l'origine d'une

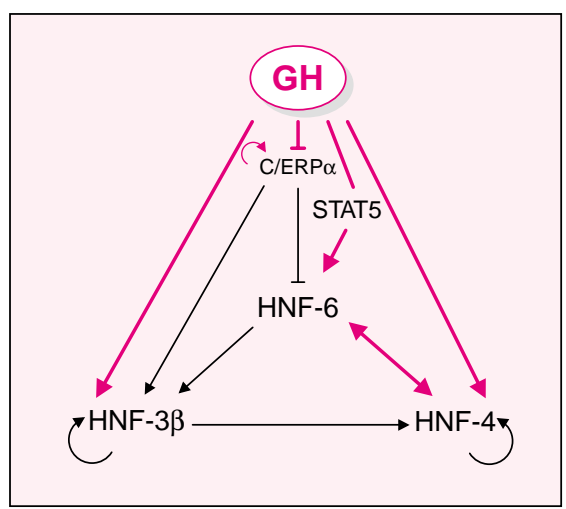

Figure 1. Rôle de l'hormone de croissance dans le contrôle d'un réseau de facteurs de transcription hépatiques. L'hormone de croissance stimule l'expression de HNF-6 par trois mécanismes : le recrutement du facteur de transcription STAT5, I'augmentation de l'affinité de la liaison de HNF-4 sur le promoteur de hnf6, et la levée de la liaison de C/EBP $\alpha$ au promoteur hnf6. De plus, l'hormone de croissance stimule l'expression de hnf3 $\beta$ par des mécanismes probablement indépendants de HNF-6. Au sein de ce réseau, HNF-6 stimule la transcription des gènes hnf3 $\beta$ et hnf4, HNF-3 $\beta$ stimule celle du gène hnf4, et HNF-4 celle du gène hfn6. $C / E B P \alpha$ inhibe la transcription $d u$ gène hnf6 mais stimule celle du gène hnf3 $\beta$. Par ailleurs, l'autorégulation positive des gènes hnf3 $\beta$, hnf4 et c/ebp $\alpha$ est bien documentée. régulation négative. Nous avons en effet montré que le promoteur du gène $h n f 6$ interagit avec un troisième facteur de transcription, $\mathrm{C} / \mathrm{EBP} \alpha$ (CCAAT/ enhancer binding protein), de la famille bZIP [8]. Alors que $\mathrm{C} / \mathrm{EBP} \alpha$ est généralement décrit comme un activateur transcriptionnel, sa liaison au promoteur du gène hnf6 inhibe fortement l'activité de celui-ci. L'effet transitoire de l'hormone de croissance sur le gène $h n f 6$ pourrait être expliqué par une action en deux temps de l'hormone sur $\mathrm{C} / \mathrm{EBP} \alpha$ : a) l'hormone de croissance provoque d'abord une chute rapide de la quantité de protéine $\mathrm{C} / \mathrm{EBP} \alpha$, permettant une diminution rapide et transitoire de la liaison de ce facteur sur le promoteur du gène $h n f 6$, et une levée de son inhibition sur la transcription du gène; b) dans un deuxième temps, on observe une réapparition de cette liaison, concomitante à l'augmentation de l'ARNm codant pour $\mathrm{C} / \mathrm{EBP} \alpha$, ce qui permet de rétablir cette inhibition [8].

\section{Un réseau complexe de facteurs} de transcription hépatiques

L'ensemble de ces données montre que HNF-6 est au centre d'un réseau de facteurs de transcription hépatiques qui interagissent via des boucles de rétroaction et d'autorégulation (figure 1). La réalité est certainement encore plus complexe, ne fût-ce qu'en raison de la présence, dans le foie, d'autres facteurs de la classe ONECUT, tels que OC-2. Ce dernier contrôle en effet l'expression de certains des gènes cibles de HNF6 comme $h n f 3 \beta$ [4]. Ce réseau de facteurs de transcription est lui-même contrôlé par l'hormone de croissance qui stimule l'expression du gène hnf6 par plusieurs mécanismes: le recrutement du facteur de transcription STAT5, l'augmentation de l'affinité de la liaison de HNF-4 sur le promoteur de $h n f 6$, et la levée de la liaison de $\mathrm{C} / \mathrm{EBP} \alpha$ au promoteur hnf6. En outre, l'hormone de croissance stimule directement HNF-3 $\beta$ et HNF-4. Dès lors qu'un stimulus, comme celui provoqué par l'hormone de croissance, vient perturber l'équilibre d'un tel réseau en agissant aussi bien sur les protéines qui en font partie que sur les gènes correspondants, on comprend bien la difficulté d'en prédire les conséquences, en raison des différences de demi-vie de tous les acteurs et des boucles de rétroaction.

Cette notion de réseau de facteurs de transcription amène à reconsidérer le mécanisme d'action de l'hormone de croissance dans le foie. Bien que la découverte de l'axe hormone de croissance-IGF-I date des années 1960, le mécanisme par lequel l'hormone de croissance stimule l'expression du gène IGF-I n'a toujours pas été clarifié. Un rôle du réseau décrit ici n'est pas à exclure. En effet, des données récentes [9] suggèrent que le facteur HNF-1 $\alpha$ est impliqué, et l'on sait que l'expression de $h n f l \alpha$ est stimulée par HNF-3ß et HNF-4. Cependant, il semble que la concentration hépatique de HNF-1 $\alpha$ ne soit pas influencée par l'hormone de croissance. Quant aux autres gènes hépatiques contrôlés par l'hormone de croissance, ceux-ci restent à identifier, et pourraient n'être que des cibles indirectes de cette hormone, dès lors que l'hormone de croissance s'impose comme chef d'orchestre de ces facteurs de transcription susceptibles chacun de modifier l'expression d'un grand nombre de gènes. L'existence d'un tel réseau et de son contrôle hormonal met à jour une réalité fort éloignée de la notion simpliste de cascades de signalisation linéaires et unidirectionnelles. Elle apporte de l'eau au moulin de l'eukaryotic synexpression group theory proposée en décembre dernier par Niehrs et Pollet dans la revue Nature [10].

1. Lahuna O, Leandro F, Karlsson H, et al. Expression of hepatocyte nuclear factor 6 in rat liver is sex-dependent and regulated by growth hormone. Proc Natl Acad Sci USA 1997 ; 94: 12309-13. 2. Lemaigre FP, Durviaux SM, Truong O, Lannoy VJ, Hsuan JJ, Rousseau GG. Hepatocyte nuclear factor 6 , a transcription factor that contains a novel type of homeodomain and a single cut domain. Proc Natl Acad Sci USA 1996 ; 93: 9460-4.

3. Lannoy VJ, Bürglin TR, Rousseau GG, Lemaigre FP. Isoforms of hepatocyte nuclear factor-6 differ in DNA-binding properties, contain a bifunctional homeodomain, and define the new onecut class of homeodomain proteins. J Biol Chem 1998 ; 273 : 13552-62. 
4. Jacquemin P, Lannoy VJ, Rousseau GG Lemaigre FP. OC-2, a novel mammalian member of the onecut class of homeodomain transcription factors whose function in liver partially overlaps with that of hepatocyte nuclear factor-6. J Biol Chem 1999 ; 274: 2665-71.

5. Pierreux CE, Stafford J, Demonte D, et al. Antiglucocorticoid activity of hepatocyte nuclear factor-6. Proc Natl Acad Sci USA 1999 ; 96: 8961-6. 6. Landry C, Clotman F, Hioki T, et al. HNF-6 is expressed in endoderm derivatives and nervous system of the mouse embryo and participates to the cross-regulatory network of liver-enriched transcription factors. Dev Biol 1997 ; 192: 247-57. 7. Lahuna O, Rastegar M, Maiter D, Thissen JP, Lemaigre FP, Rousseau GG. Involvement of STAT5 (signal transducer and activator of transcription 5) and HNF-4 (hepatocyte nuclear factor 4) in the transcriptional control of the $h n f 6$ gene by growth hormone. Mol Endocrinol 2000 ; 14: 285-94.

8. Rastegar M, Rousseau GG, Lemaigre FP. CCAAT/Enhancer binding protein alpha $\mathrm{C} / \mathrm{EBP} \alpha)$ is a component of the growth hormone-related network of liver transcription factors. Endocrinology $2000 ; 141: 1686-92$ 9. Meton I, Boot EPJ, Sussenbach JS, Steenbergh PH. Growth hormone induces insulin-like growth factor-I gene transcription by a synergistic . 444: 155-9.

10. Niehrs C, Pollet N. Synexpression groups in eukaryotes. Nature 1999 ; 402 : 483-7.

\section{Mojgan Rastegar \\ Frédéric P. Lemaigre \\ Guy G. Rousseau}

Unité hormones et métabolisme, Université catholique de Louvain et Christian de Duve, Institute of Cellular Pathology, 75, avenue Hippocrate, B-1200, Bruxelles, Belgique.
Pancréas endocrine: HNF-6 au sommet de la hiérarchie ? Le pancréas contient trois types de cellules: les cellules endocrines produisent de l'insuline, du glucagon, de la somatostatine, ou du polypeptide pancréatique; les cellules exocrines sécrètent des enzymes digestives, et les cellules canalaires permettent la sécrétion de ces enzymes. Alors que tous ces types cellulaires dérivent d'un même épithélium issu du tube digestif embryonnaire, on ignore les facteurs qui déterminent leur différenciation. Des résultats récents plaident pour un mécanisme de "spécification latérale", comme c'est le cas dans le système nerveux. Une cellule qui développe un programme de différenciation donné (grâce à un facteur de transcription appelé neurogénine) supprime ce même programme chez sa voisine en y réprimant le gène de la neurogénine. Pour ce faire, la cellule « inhibitrice» produit un ligand qui active le récepteur «Notch» porté par cette voisine. Dans le pancréas de souris dont le système "Notch » a été invalidé ou dans lequel la neurogénine-3 (Ngn-3) est surexprimée, la différenciation se fait préférentiellement vers la voie endocrine [1]. De plus, il n'y a pas de cellules endocrines en cas d'invalidation du gène $n g n 3$ [2]. Cette donnée faisait de Ngn-3 le facteur de transcription le plus en amont dans la chaîne de décision menant à la différenciation endocrine. Elle posait aussi la question de l'identité du facteur qui stimule l'expression de ngn3. Un candidat sérieux est HNF-6 ( $\mathrm{m} / \mathrm{s} 1996$, $n^{\circ} 12, p .1431$ ), le prototype de la classe "ONECUT» des facteurs de transcription [3]. Des travaux récents du groupe de Guy Rousseau et Frédéric Lemaigre montrent que les cellules du pancréas embryonnaire qui expriment ngn3 expriment également $h n f 6$, et que le promoteur ngn3 lie HNF-6. De plus, la transfection de HNF-6 dans des cel- lules en culture y stimule la transcription de ngn3. Conformément à ces résultats, l'invalidation du gène hnf6 chez la souris abolit quasi complètement l'expression pancréatique de $n g n 3$ [4], et se solde par un grave défaut de différenciation endocrine [4]. Ainsi, HNF-6 serait le premier régulateur positif connu du gène "pro-endocrine" ngn3. La découverte de ce rôle de HNF-6, et la compréhension des mécanismes qui conduisent du précurseur pluripotent à la cellule $\beta$ productrice d'insuline, devraient accélérer l'émergence d'une thérapie cellulaire du diabète.

[1. Apelqvist A, et al. Nature 1999; 400: 877-81.]

[2. Gradwohl G, et al. Proc Natl Acad Sci USA 2000; 97 : 1607-11.]

[3. Lannoy VJ, et al. I Biol Chem 1998; 273: 13552-62.]

[4. Jacquemin P, et al. Mol Cell Biol $2000 ; 20: 4445-54$.

\footnotetext{
Ouverture, à la rentrée Universitaire 2000, d'un Diplôme d'Études Spécialisée Complémentaires (DESC) en addictologie

Une mesure du plan triennal

Dans le cadre du plan triennal de lutte contre la drogue et de prévention des dépendances, adopté par le Gouvernement le 16 juin 1999, un certain nombre de mesures visaient à améliorer la formation initiale et continue des médecins.

En effet, il est nécessaire de permettre aux personnes ayant acquis des compétences en toxicomanie, en alcoologie et en tabacologie d'avoir une reconnaissance universitaire.

Comment il se déroule?

Il est mis en place sur deux ans et comporte deux volets de formation :

- une "théorique" (120 heures d'enseignement) constituée de 6 modules (problématique générale, santé publique : aspects sociaux

et législatifs, approche spécifique des addictions, les conduites à tenir),

- une "pratique " comportant 4 semestres de stages validants.

Comment s'inscrire?

Chaque interne, médecin ou spécialiste qui souhaite s'inscrire est invité à prendre contact au secrétariat du $3^{\text {e }}$ cycle de sa faculté de

Médecine d'origine avant le $1^{\text {er }}$ décembre 2000.

Contact presse : MILDT - Patrick Chanson - Tél. : 0140566288.
} 